Technical Report

\title{
Utilizing novel telepathology system in preclinical studies and peer review
}

\author{
Gabriel Siegel ${ }^{1}$, Dan Regelman 1 , Robert Maronpot ${ }^{2}$, Moti Rosenstock ${ }^{3}$, Shim-mo Hayashi ${ }^{4}$, and \\ Abraham Nyska ${ }^{5,6^{*}}$
}

\author{
${ }^{1}$ Augmentiqs, LIAD building, P.O. Box 10, Techelet Street, Teradion Business Park - D.N. Misgav 2017400, Israel \\ 2 Maronpot Consulting, LLC, 1612 Medfield Road, Raleigh, North Carolina 27607, USA \\ ${ }^{3}$ LEA, Nonclinical Safety Consultancy, Talmei Elazar, 38812, Israel \\ ${ }^{4}$ Global Scientific and Regulatory Affairs, San-Ei Gen, F.F.I., Inc., 1-1-11 Sanwa-cho, Toyonaka, Osaka 561-8588, Japan \\ ${ }^{5}$ Sackler School of Medicine, Tel Aviv University, P.O. Box 39040, Tel Aviv 6997801, Israel \\ ${ }^{6}$ Toxicologic Pathology, Haharuv 18, P.O. Box 184, Timrat 36576, Israel
}

\begin{abstract}
The demand for board certified toxicologic pathologists and expansion of IT capabilities has resulted in an increase of "virtual" and "distance" pathology (telepathology), and a need for greater flexibility in real time consultations. Newly developed microscope-integrated telepathology systems enable geographically remote stakeholders to view the live histopathology slide as seen by the microscope user. Simultaneous online viewing, annotation, and dialog between pathologists and study experts facilitates good science and economic benefits by enabling more timely and informed clinical decisions. (DOI: 10.1293/tox.2018-0032; J Toxicol Pathol 2018; 31: 315-319)
\end{abstract}

Key words: telepathology, toxicology, toxicologic pathology

Recent advances in real-time telepathology are enhancing the manner in which toxicology studies are being conducted; enabling in-depth collaboration among study experts and pathologists, faster and more informed decision making, and greater understanding of the pathology process among all stakeholders.

One such technology called Microscope Integrated Telepathology (MIT) was lately developed by Augmentiqs. It is a novel system which enhances the functionality of existing infinity-corrected light microscopes, enabling the microscope operator to instantly share and discuss the live image of the tissue sample with multiple remote parties, whom are able to simultaneously view and annotate the image from their computer ${ }^{1}$.

This approach to telepathology is cost-efficient and enhances not only preclinical and toxicologic pathology studies, but also diagnostic consulting, biomedical research and training. Furthermore, it offers advantages in workflow and collaboration that are not available in existing telepathology

Received: 19 May 2018, Accepted: 20 June 2018

Published online in J-STAGE: 8 July 2018

*Corresponding author: A Nyska (e-mail: anyska@bezeqint.net) (C)2018 The Japanese Society of Toxicologic Pathology

This is an open-access article distributed under the terms of the Creative Commons Attribution Non-Commercial No Derivatives (c) $(1)$ (by-nc-nd) License. (CC-BY-NC-ND 4.0: https:// (c) 1 BY NC $\bigoplus_{\text {ND }}$ creativecommons.org/licenses/by-nc-nd/4.0/). technologies.

\section{The Current State of Telepathology}

Telepathology is used globally and is considered invaluable in toxicologic pathology ${ }^{2}$. It has traditionally been associated with Whole Slide Imaging (WSI), a technology that creates a permanent digital copy of the glass slide at multiple magnifications, and enables distant parties to view the information, select image fields for photography, and collect in-depth image analysis data in addition to simple measurements ${ }^{3}$. Along with the clear advantages of WSI for creating digital data from glass slides, when attempting to utilize WSI for real-time telepathology applications, it is found that WSI does not lend well for creating an environment of interactive discussion and collaboration between multiple remote pathologists.

Typical viewing of whole scanned slides is performed as a single user, and not as a simultaneous dynamic discussion involving the pathologist, the sponsor and multiple remote experts. When comparing WSI to the viewing of slides under a microscope, the time required for scanning slides is high, while the digitally heavy files of the entire slide are not easily transferred to remote parties. Furthermore, WSI typically represents a single flat plane of focus, and is associated with a high capital expense with ongoing maintenance costs.

All the above substantially reduce the utility and effectiveness of WSI when compared to a physically co-lo- 
cated discussion using a multi-headed microscope. Since the pathologist's primary tool is still the microscope, an effective means for real time and dynamic sharing of a high quality live view of the microscope's optical field is the basic requirement for any telepathology application. Table 1 provides a comparison between the MIT System and other well-known digital technologies.

\section{The Introduction of Microscope-integrated Tele- pathology (MIT)}

With the introduction of MIT systems that we present here, there is the technology to share a high-quality, comparable and live view of the microscope's optical plane with remote study experts and stakeholders. The technology enables multi-directional collaboration among an unlimited number of remote parties, in real-time, and without the use of scanning - similar in concept to a virtual double-headed scope.

The method in which the new MIT telepathology technology works is depicted in Fig. 1. A small Optical Module becomes an integrated component of any existing infinitycorrected light microscope by being placed in the optical path above the nose piece and below the eyepieces. The microscope user continues as before to view the tissue through the eyepiece, while an embedded image sensor within the Optical Module captures a live feed of the tissue that is shared with remote parties over a dedicated communications protocol without image loss.

The Optical Module further enhances pathology workflow by projecting an augmented overlay of digital information on top of the optical field of view within the microscope eyepiece. The microscope user may perform annotations, morphometric calculations or compare digital images to the existing slide, such as making animal to animal comparisons during the slide evaluation phase, while all the time looking within the microscope eyepiece. These annotations

Table 1. Comparison between the MIT System and Other Wellknown Digital Technologies

\begin{tabular}{lccc}
\hline & $\begin{array}{c}\text { Integrated } \\
\text { Telepathology }\end{array}$ & $\begin{array}{c}\text { 3rd } \\
\text { Ocular } \\
\text { Camera }\end{array}$ & $\begin{array}{c}\text { Whole } \\
\text { Slide } \\
\text { Imaging }\end{array}$ \\
\hline Capital Expenditure & Low & Low & High \\
Ongoing Maintenance & Low & Low & High \\
Regulatory Requirements & Low & Low & High \\
Microscope Integration & Yes & Yes & No \\
Augmented Microscope Eyepiece & Yes & No & No \\
Real-Time Telepathology & Yes & No & No \\
Field of View & Equal to & Narrow & Full Slide \\
Image File Size & Eyepiece & \multicolumn{3}{c}{ Mb Up to $2 \mathrm{~Gb}$} \\
Workflow Enhancement & $2-5 \mathrm{Mb}$ & $2-5 \mathrm{Mb}$ & No \\
\hline
\end{tabular}

Copied with permission from: Siegel S, Regelman R, Maronpot RR, Rosenstock M, Nyska A (2017). New technologies: real time telepathology systems - novel cost-effective tools for real-time consultation and data sharing. Toxicologic Pathology 45(8): 1039-1042. and measurements include not only tools native to the MIT system, but rather the MIT system can act as a platform for 3rd party software such as Image $J$ and other pathology software. All actions performed are automatically shared and visible by all remote participants.

During a telepathology session, remote viewers may connect to the user's microscope by downloading a client software that displays high-resolution images regardless of Internet latency. The microscope user may optionally work on an adjacent computer screen attached to the computer and see exactly the same view as all other group participants.

During the telepathology session, the remote viewers talks to the microscope user over their regular telephone or any other digital voice communication means, and may perform annotations, morphometric calculations and download TIFF or PNG images directly to their personal computer, while the microscope user can work the mechanical stage and change objectives for increased magnification as necessary.

\section{Recent Application of Telepathology in Toxico- logic Pathology}

Pathology is a subjective science, and within toxicologic studies there exists many potential sources of inconsistency in diagnosing lesions ${ }^{4}$. For this reason, pathology peer review is commonly being performed to increase confidence that treatment-related findings are properly identified, consistently diagnosed, and correctly interpreted ${ }^{5}$. While face-to-face interaction over a multiheaded microscope is considered the ideal methodology for peer review, it could be conducted remotely if remote parties can effectively eval-

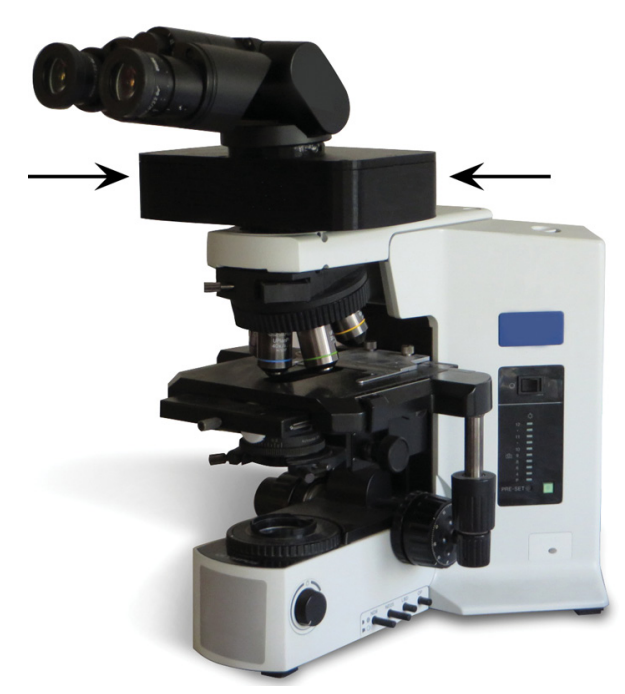

Fig. 1. Optical Module (arrows) installed on any existing light microscope. Copied with permission from: Siegel S, Regelman R, Maronpot RR, Rosenstock M, Nyska A (2017). New technologies: real time telepathology systems-novel cost-effective tools for real-time consultation and data sharing. Toxicologic Pathology 45(8): 1039-1042. 
uate tissue changes via digital images of the glass slide in real time 6 .

As an example application of MIT technology, a remote online peer review using the above-mentioned MIT technology was recently conducted involving a number of the authors. The peer review pathologist (PRP) located in Timrat, Israel received several thousand slides stemming from a preclinical study conducted on behalf of a non-U.S.based drug developer from a contract research organization located in the United States. After initial review, the PRP set aside a number of the slides for further review with the study pathologist (SP) due to a difference in opinion regard- ing the classification of the lesions. The online peer review included the PRP and SP, and enabled a dynamic discussion revolving around a real-time view of the slides on the microscope stage. Both the PRP and SP were able to make annotations, select regions of interest, conduct morphometric analysis and download images directly to document the peer review event. Fig. 2A-D are demonstrating 2 examples of recently application of the MIT technology.

Upon completion of such a slide review, the PRP may elect to prepare a "Live Telepathology Review Report" to document the peer review procedure, and potentially may include representative photomicrographs of the slides that

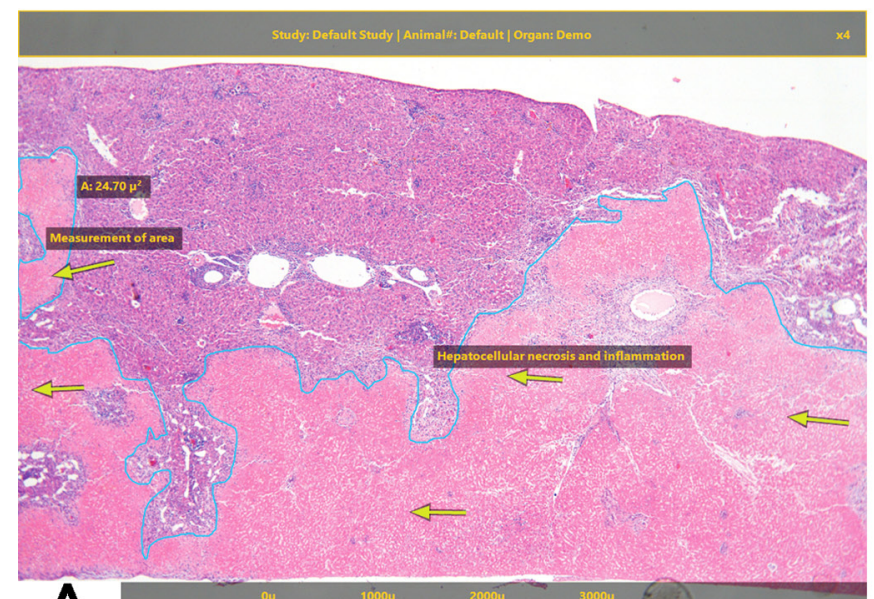

A
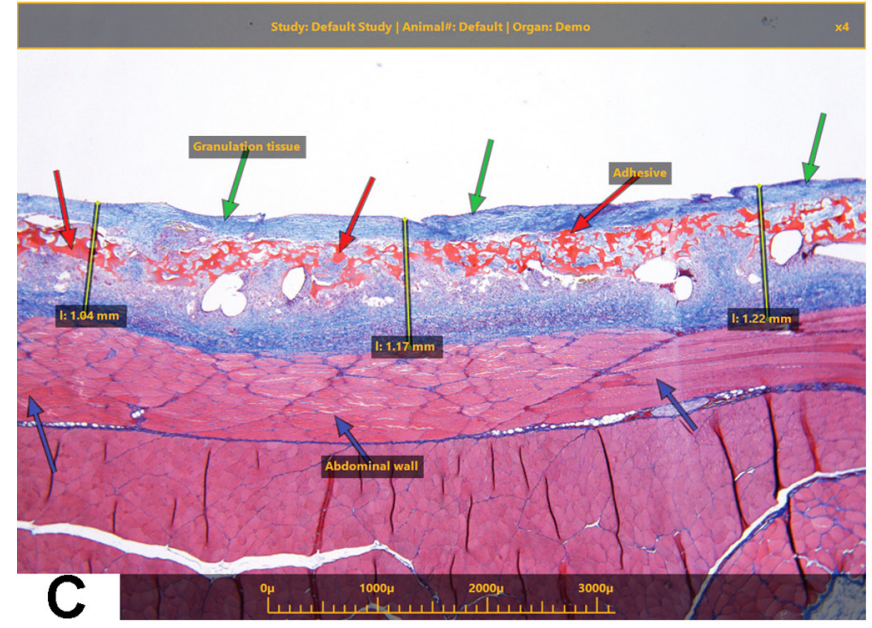
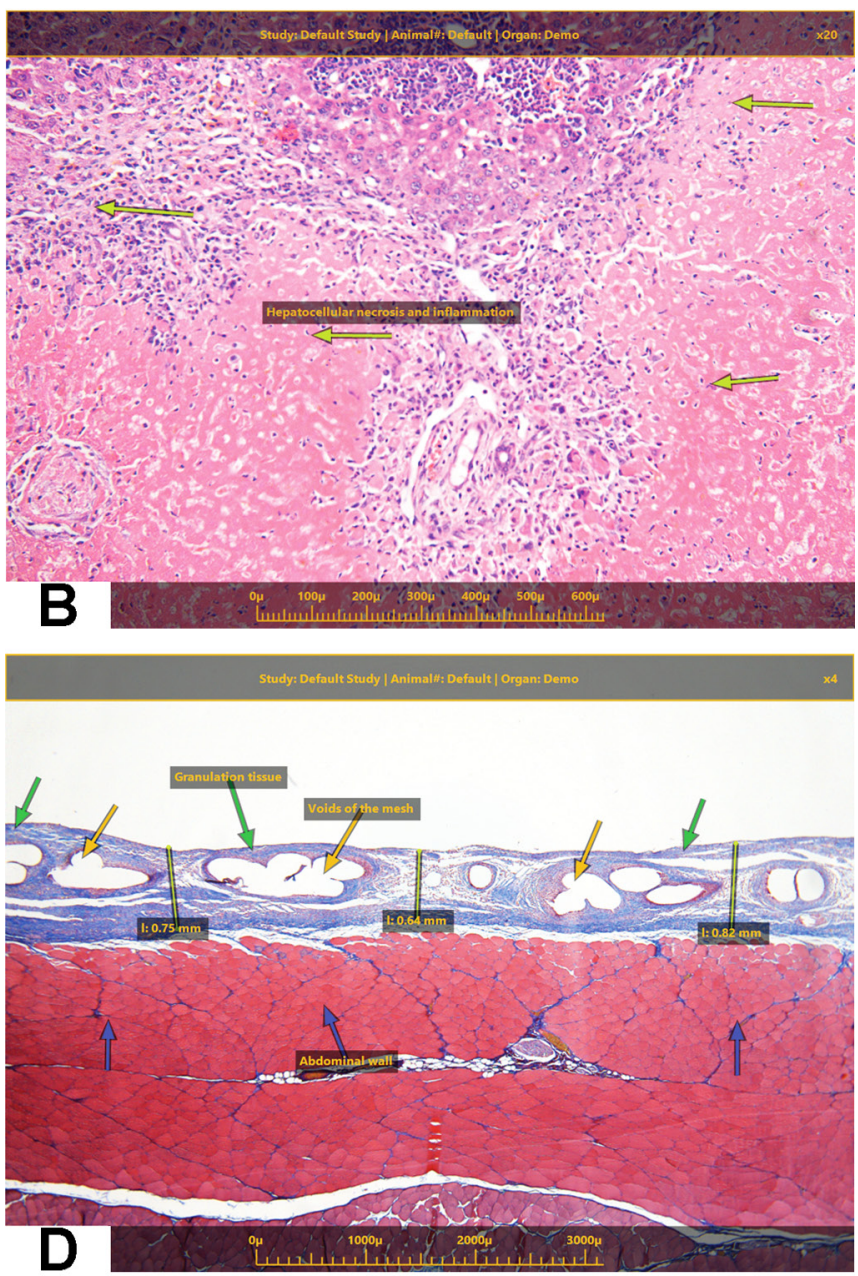

Fig. 2. (A and B) A recently conducted study with an objective of assessing the safety of an anti-cancer therapeutic modality in mice, following intravenous (IV) single administration was performed. Histopathological evaluation indicated extensive hepatocellular necrosis and inflammation (A and B). The live telepathology technology helped in instantly sharing and documentation of the microscopic findings with the study director and the sponsor both adding annotations and measurements (i.e., area of the necrosis) from their computer. (C and D) The objective of this second study was to assess the biodegradability and local effects of the Test Item, a medical device with a biodegradable (C) and non-degradable component (D), following intra-peritoneal (IP) implantation on the abdominal wall in SD rats over the course of 2 weeks. The tissue reaction related to the presence of the biodegradable component $(\mathrm{C})$ is characterized by the presence of mild granulation tissue, which was of uniform thickness along the entire implantation site, and intimately surrounded also the device. The tissue reaction related to the presence of the Reference device (i.e., without non-degradable component) (D) is characterized by the presence of mild granulation tissue, which was of not uniform thickness along the entire implantation site. The live telepathology technology helped in instantly sharing and documentation of the microscopic findings with the study director and the sponsor, both of whom added annotations and measurements (i.e., thickness of the granulation tissue) from their computer. 
were shared. Such an unofficial report could serve as study notes shared among the SP, PRP, study director, and other stakeholders. Ultimately, the SP and PRP signed a Formal Peer Review Report signifying achievement of diagnostic consensus during the telepathology session. This document is eventually included in a formal study report suitable for any regulatory submission.

\section{Significance of MIT Technology}

In addition to improving the science of toxicology studies, the significance of this methodology for online telepathology is significant for its quality as well as cost and time savings.

The cost benefits of the novel MIT system can be appreciated from several vantage points. Installation and use of the system is minimal, making it an easy accessory for any pathologist to integrate within their existing microscope. Furthermore, the benefit of reducing travel and the logistics of slide shipment can easily reach many thousands of dollars per study. However, the largest cost benefit may be the time saved for the sponsor - allowing them to reach business decisions faster. And in particular to the abovementioned example where the SP at the contract research organization labeled certain lesions, and subsequently during the telepathology session with the PRP changed these definitions, a consensus was reached that effectively and efficiently completed the pathology peer review and allowed the toxicology report to be finalized.

\section{Future Directions}

As toxicology continues to be an even more global endeavor, the need for greater remote collaboration and effective communications will grow in scope, as will the need for new technologies which offer cost and time saving benefits. As MIT technology is essentially the ability to turn the existing microscope into a virtual multi-headed scope with digital capabilities, there exists the possibility for new applications in toxicology; including new techniques for nomenclature standardization and incorporation of tools for more streamlined reporting. The MIT system mentioned above can act as a platform for running other pathology software, enabling pathologists to utilize their existing digital toolsets within the classic workflow. In addition, the capability of the MIT system to efficiently capture high resolution, whitebalanced photomicrographs serve as an alternative camera that can be used on the microscope for routinely acquiring photomicrographs.

MIT can also play a significant role in allowing regulatory agencies a peek into the decision-making process of new drugs, or at the very least present a new method for sharing findings.

As the new MIT technology becomes more commonly used, researchers will find and examine new capabilities, such as use in histopathology training programs, for the existing microscope.

\section{Conclusions}

In conclusion, MIT telepathology systems enables cost-effective and practical collaboration in biomedical research and preclinical studies without necessitating travel of pathology experts or extensive shipping of slides (Fig. 3). It can have a positive impact on commercial activities of study sponsors by facilitating informed decision making relating to study results in a shorter period of time. In addition, MIT facilitates good science by supporting informal and formal consultation and knowledge sharing within the framework of existing best practices for toxicology sciences.

Disclosure of Potential Conflicts of Interest: G.S. and D.R. are employees and/or consultants of Augmentiqs. A.N. serves as a consultant for Augmentiqs. R.M. serves as an advisor and champion of new technologies that have toxicologic pathology applications.

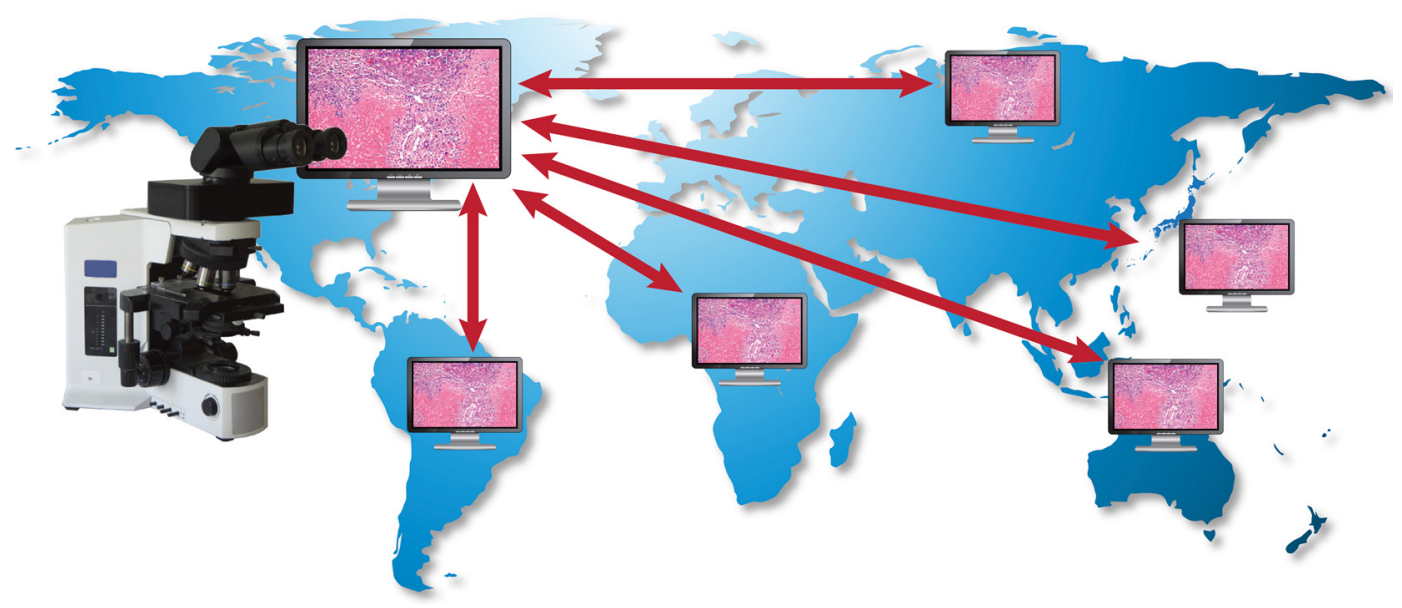

Fig. 3. Globalization in preclinical toxicity and pharmacology studies - Diagram demonstrating the use of Microscope Integrated Telepathology. 


\section{References}

1. Siegel G, Regelman D, Maronpot R, Rosenstock M, and Nyska A. New technologies: Real-time telepathology systems-novel cost-effective tools for real-time consultation and data sharing. Toxicol Pathol. 45: 1039-1042. 2017. [Medline] [CrossRef]

2. Ettlin RA. Toxicologic pathology in the 21st century. Toxicol Pathol. 41: 689-708. 2013. [Medline] [CrossRef]

3. Hanna MG, Pantanowitz L, and Evans AJ. Overview of contemporary guidelines in digital pathology: what is available in 2015 and what still needs to be addressed? J Clin Pathol. 68: 499-505. 2015. [Medline] [CrossRef]
4. Cesta MF, Malarkey DE, Herbert RA, Brix A, Hamlin MH 2nd, Singletary E, Sills RC, Bucher JR, and Birnbaum LS. The National Toxicology Program Web-based nonneoplastic lesion atlas: a global toxicology and pathology resource. Toxicol Pathol. 42: 458-460. 2014. [Medline] [CrossRef]

5. Crissman JW, Goodman DG, Hildebrandt PK, Maronpot RR, Prater DA, Riley JH, Seaman WJ, and Thake DC. Best practices guideline: toxicologic histopathology. Toxicol Pathol. 32: 126-131. 2004. [Medline] [CrossRef]

6. Morton D, Sellers RS, Barale-Thomas E, Bolon B, George C, Hardisty JF, Irizarry A, McKay JS, Odin M, and Teranishi M. Recommendations for pathology peer review. Toxicol Pathol. 38: 1118-1127. 2010. [Medline] [CrossRef] 